\title{
Compliant Floating-Base Control of Space Robots
}

\author{
Alessandro M. Giordano ${ }^{(\circledR)}$, Davide Calzolari ${ }^{\circledR}$, Marco De Stefano ${ }^{\circledR}$, Hrishik Mishra ${ }^{\circledR}$, \\ Christian Ott ${ }^{\circledR}$, Senior Member, IEEE, and Alin Albu-Schäffer ${ }^{\circledR}$, Fellow, IEEE
}

\begin{abstract}
This letter presents a compliant feedback controller of an arm-equipped spacecraft, which does not enforce requirements on the spacecraft position and attitude. The controller is applicable to the pre-contact, contact, and post-contact phases of a robotic operation. In contrast to conventional floating-base strategies, the controller eliminates the instability of the system during a steady contact, and thus can be used in realistic applications. The controller uses an external-internal transposed-Jacobian control for compliant regulation of the end-effector, together with regulation of the whole-body Center-of-Mass (CoM) and angular momentum for achieving post-contact stability, and force feedback for achieving a stable contact phase. The method is validated experimentally using a hardware-in-the-loop simulator composed of a seven degrees-offreedom (DOF) arm mounted on a 6 DOF simulated spacecraft. Numerical simulations further validate the method considering a realistic thrusters system, measurement noise, and time delay.
\end{abstract}

Index Terms-Space robotics and automation, compliance and impedance control, passivity, motion control, dynamics.

\section{INTRODUCTION}

$\mathbf{T}$ HE capture of a target in orbit by means of space robot, i.e., a spacecraft equipped with an arm, requires a controller capable of ensuring a safe contact phase. Contact may cause damage to the robot or to the target; it shall be ensured that the interaction takes place in a compliant way, i.e., such that deviations of the end effector due to external forces are allowed and, in turn, the contact forces are kept within acceptable limits. The focus of this letter is the compliant control of space robots without control requirements on the spacecraft pose, i.e., position and attitude, which herein will be simply referred to as compliant floating-base control. The possibility of leaving free the degrees of freedom (DOF) of the spacecraft during the maneuvering of the arm is envisioned in some missions [1], [2], because it allows reducing the limitations induced by the thrusters system, e.g., low-rate on/off actuation and consumption of nonrenewable fuel.

Manuscript received February 24, 2021; accepted June 27, 2021. Date of publication July 16, 2021; date of current version August 3, 2021. This letter was recommended for publication by Associate Editor J. Cacace and Editor P. Pounds upon evaluation of the reviewers' comments. (Corresponding author: Alessandro M. Giordano.)

Alessandro M. Giordano, Davide Calzolari, and Alin Albu-Schäffer are with the Institute of Robotics and Mechatronics, German Aerospace Center (DLR), 82234 Weßling, Germany, and also with the Department of Informatics, Technische Universität München (TUM), 85748 Garching, Germany (e-mail: Alessandro.Giordano@dlr.de; davide.calzolari@dlr.de; Alin.Albu-Schaeffer@dlr.de).

Marco De Stefano, Hrishik Mishra, and Christian Ott are with the Institute of Robotics and Mechatronics, German Aerospace Center (DLR), 82234 Weßling, Germany (e-mail: marco.destefano@dlr.de; Hrishik.Mishra@dlr.de; christian.ott@dlr.de).

This letter has supplementary downloadable material available at https://doi. org/10.1109/LRA.2021.3097496, provided by the authors

Digital Object Identifier 10.1109/LRA.2021.3097496
The problem of contact between a space robot and a target in orbit has been addressed in several works [3]-[11]. In [3] the impact dynamics between a floating multibody chain and a rigid body was modelled based on the conservation of momentum. In [4], [6] the momentum redistribution for the grasping of a tumbling satellite was investigated. In [7] the drift ${ }^{1}$ of a taskspace-controlled free-floating robot due to nonzero momentum during post-contact phase was pointed out. In [5] the criticalities of open contact with a floating target were discussed. In [5], [9], [11] tuning conditions for achieving velocity matching of end-effector and target in contact were proposed. In the field of floating-base compliant control, purely free-floating controllers have been employed in [2], [5] and [1], while actuated-base controllers have been employed in [7], [8] and [12]. In the pioneering work [1], a transposed-Jacobian free-floating position controller was proposed, which in fact results being an impedance controller when in contact; in [5] a feedback-linearization freefloating impedance controller was proposed; in [2], a compliant tracking free-floating controller based on a visual servoing loop was proposed. In [12], a free-floating controller and a controller featuring spacecraft regulation were used via control switching; the spacecraft regulation was employed to keep the base within a prescribed distance from the target. In [7], [8] single continuous controllers were proposed for the compliant regulation of the end effector while simultaneously regulating the whole-body CoM and angular momentum.

The above-mentioned controllers are, however, affected by limitations which may impede applications in real scenarios. In totally underactuated strategies such as [1], [2], [5], the problem of the compliant control of the end effector was addressed, but the stability of rest of the system during contact was not; in fact, in such strategies the rest of the system is unstable [12] during contact, and drifts [7], [12] after contact; therefore, they are risky to use. The switching-based strategy in [12] partially addressed the problem by regulation of the distance between spacecraft and target. However, the controller is still unstable during contact and drifts after contact, because the regulation of the distance contributes to only one DOF, and the robot is still underactuated in two translational DOFs and three rotational DOFs. For general contact directions, all DOFs get excited and thus the strategy cannot be applied. This problem was solved considering short contact by the strategy [7], [8] via stabilization of all six DOFs. However, the controllers developed in [7], [8] cannot be used with prolonged contact. In particular, their limitation is that during prolonged contact the whole-body CoM error and angular momentum converge to nonzero values. The nonzero steady state errors result in a net displacement of the CoM and a drift of the attitude of the spacecraft during contact. The attitude drift is dangerous because singularities, self-collisions, or joint limits

\footnotetext{
${ }^{1}$ By "drift" we mean an unbounded growth of some configuration state(s) of the system, happening at a bounded rate.
} 


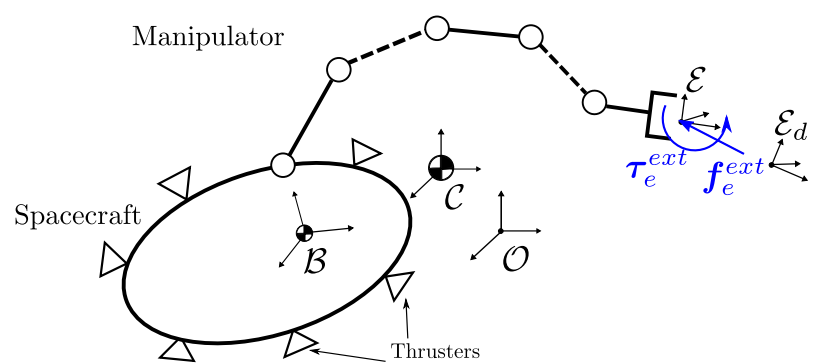

Fig. 1. Schematic representation of the system: arm-equipped spacecraft subject to contact force $\boldsymbol{f}_{e}^{e x t}$ and contact torque $\tau_{e}^{e x t}$ during the reach of a desired pose $\mathcal{E}_{d}$

may be reached during the end-effector operation. The CoM displacement contributes to the same risks, further jeopardizing the end-effector task.

In this letter we propose a compliant floating-base controller that has no drift during general contact, i.e., short or prolonged contact, and thus is applicable for manipulation. The controller exploits an external-internal transposed-Jacobian approach [7] for achieving compliant and thrusters-efficient regulation of the end-effector. Further, it employs force feedback in the external subsystem in combination with regulation of the whole-body CoM and angular momentum, for achieving the stabilization in presence of prolonged contact. The main contributions are: the derivation of a compliant floating-base controller, which has no drift and uses ideally no thrusters during a pre-contact phase; the numerical validation of the controller considering realistic models of thrusters and noisy measurements; the experimental validation of the controller during the phases of floating-base pre-contact maneuvering and steady contact.

The letter is structured as follows: Section II introduces the main equations; Section III derives the proposed controller; Section IV and Section V show the numerical and experimental validation, respectively; Section VI discusses the limitations; Section VII draws the main conclusions.

\section{PRELIMINARIES}

A space robot is considered, which is composed of a spacecraft equipped with a serial-link manipulator with $n$ revolute joints. The spacecraft is fully actuated, i.e., external forces and torques are exerted on the spacecraft by means of thrusters. The scenario involves the maneuvering of the robot's end-effector with no control requirements on the spacecraft's position and orientation, and in presence of contact. The problem developed herein is the derivation of a controller that

(R1) regulates the pose of the end effector compliantly,

(R2) can sustain a prolonged contact,

(R3) does not regulate the pose of the spacecraft and ideally uses no thrusters during a pre-contact phase.

Fig. 1 shows a schematic representation of the system components. The following frames are considered: a base frame $\mathcal{B}$ attached to the spacecraft and placed on its CoM, an end-effector frame $\mathcal{E}$, a desired end-effector frame $\mathcal{E}_{d}$, a frame $\mathcal{C}$ placed on the CoM of the space robot and whose axes are inertial, and an orbital frame $\mathcal{O}$ coincident with $\mathcal{C}$ at the beginning of the robotic operations. No orbital environmental disturbances are considered for the definition of the controller because they are considerably smaller than the actuation forces; thus the frame $\mathcal{O}$ is considered inertial.

\section{A. Notation}

The symbol $\mathcal{F}_{b}=\left[\begin{array}{ll}\boldsymbol{f}_{b}^{T} & \boldsymbol{\tau}_{b}^{T}\end{array}\right]^{T} \in \mathbb{R}^{6}$ denotes the wrench about $\mathcal{B}$ acted upon by the thrusters and expressed in the frame $\mathcal{B}$, with $\boldsymbol{f}_{b} \in \mathbb{R}^{3}$ and $\boldsymbol{\tau}_{b} \in \mathbb{R}^{3}$ being the force and torque, respectively. Let us indicate [13] with $\boldsymbol{o}_{x y} \in \mathbb{R}^{3}, \boldsymbol{R}_{x y} \in \mathrm{SO}(3)$, the position vector from a general frame $\mathcal{X}$ to a general frame $\mathcal{Y}$ expressed in the frame $\mathcal{X}$, and the corresponding rotation matrix, respectively. The use of only one superscript, i.e., $\boldsymbol{o}_{y}$ or $\boldsymbol{R}_{y}$, indicates that the quantity is relative to the inertial frame $\mathcal{O}$. The so-called Adjoint transformation [13] is used herein:

$$
\boldsymbol{A d}_{x y}=\left[\begin{array}{cc}
\boldsymbol{R}_{x y} & {\left[\boldsymbol{o}_{x y}\right]^{\times} \boldsymbol{R}_{x y}} \\
\mathbf{0} & \boldsymbol{R}_{x y}
\end{array}\right] \in \mathbb{R}^{6 \times 6},
$$

where the operator $[\cdot]^{\times}$denotes the skew-symmetric matrix of the vector argument. Let us denote as $\boldsymbol{\nu}_{y}=\left[\begin{array}{ll}\boldsymbol{v}_{y}^{T} & \boldsymbol{\omega}_{y}^{T}\end{array}\right]^{T} \in \mathbb{R}^{6}$ the body velocity of a general frame $\mathcal{Y}$, where $\boldsymbol{v}_{y} \in \mathbb{R}^{3}$ and $\boldsymbol{\omega}_{y} \in \mathbb{R}^{3}$ are its linear and angular parts. The symbol $\mathbf{0}$ denotes the zero matrix of suitable dimensions.

\section{B. Kinematics and Dynamics}

The end-effector velocity is expressed as

$$
\boldsymbol{\nu}_{e}=\boldsymbol{J}_{e}(\boldsymbol{q})\left[\begin{array}{c}
\boldsymbol{\nu}_{b} \\
\dot{\boldsymbol{q}}
\end{array}\right], \quad \boldsymbol{J}_{e}(\boldsymbol{q})=\left[\begin{array}{ll}
\boldsymbol{A d}_{e b}(\boldsymbol{q}) & \boldsymbol{J}_{e m}(\boldsymbol{q})
\end{array}\right]
$$

where $\boldsymbol{q} \in \mathbb{R}^{n}$ are the joint angles and $\boldsymbol{J}_{\text {em }}(\boldsymbol{q}) \in \mathbb{R}^{6 \times n}$ is the manipulator Jacobian matrix.

The dynamics of the space robot is described [14] by

$$
\begin{aligned}
& \underbrace{\left[\begin{array}{cc}
\boldsymbol{M}_{b} & \boldsymbol{M}_{b m} \\
\boldsymbol{M}_{b m}^{T} & \boldsymbol{M}_{m}
\end{array}\right]}_{\boldsymbol{M}(\boldsymbol{q})}\left[\begin{array}{c}
\dot{\boldsymbol{\nu}}_{b} \\
\ddot{\boldsymbol{q}}
\end{array}\right]+\underbrace{\left[\begin{array}{cc}
\boldsymbol{C}_{b} & \boldsymbol{C}_{b m} \\
\boldsymbol{C}_{m b} & \boldsymbol{C}_{m}
\end{array}\right]}_{\boldsymbol{C}\left(\boldsymbol{q}, \boldsymbol{\nu}_{b}, \dot{\boldsymbol{q}}\right)}\left[\begin{array}{c}
\boldsymbol{\nu}_{b} \\
\dot{\boldsymbol{q}}
\end{array}\right] \\
& =\left[\begin{array}{c}
\mathcal{F}_{b} \\
\boldsymbol{\tau}
\end{array}\right]+\boldsymbol{J}_{e}(\boldsymbol{q})^{T} \mathcal{F}_{e}^{e x t}
\end{aligned}
$$

where $\quad \tau \in \mathbb{R}^{n} \quad$ denotes the joint torques; $\mathcal{F}_{e}^{e x t}=\left[\begin{array}{ll}\boldsymbol{f}_{e}^{e x t T} & \boldsymbol{\tau}_{e}^{e x t T}\end{array}\right]^{T} \in \mathbb{R}^{6}$ is the contact wrench at the end effector, with $\boldsymbol{f}_{e}^{e x t} \in \mathbb{R}^{3}$ and $\tau_{e}^{e x t} \in \mathbb{R}^{3}$ being its force and torque components, respectively; $\boldsymbol{M}(\boldsymbol{q}) \in \mathbb{R}^{(6+n) \times(6+n)}$ and $\boldsymbol{C}\left(\boldsymbol{q}, \boldsymbol{\nu}_{b}, \dot{\boldsymbol{q}}\right) \in \mathbb{R}^{(6+n) \times(6+n)}$ are the inertia and Coriolis/centrifugal matrices, respectively, for which it holds $\dot{\boldsymbol{M}}(\boldsymbol{q}, \dot{\boldsymbol{q}})=\boldsymbol{C}\left(\boldsymbol{q}, \boldsymbol{\nu}_{b}, \dot{\boldsymbol{q}}\right)+\boldsymbol{C}\left(\boldsymbol{q}, \boldsymbol{\nu}_{b}, \dot{\boldsymbol{q}}\right)^{T}$

The momentum $\boldsymbol{h}_{c} \in \mathbb{R}^{6}$ of the whole system around the CoM, and the CoM velocity $\boldsymbol{v}_{c} \in \mathbb{R}^{3}$, are given by

$$
\boldsymbol{h}_{c}=\left[\begin{array}{l}
\boldsymbol{p} \\
\boldsymbol{l}_{c}
\end{array}\right] \in \mathbb{R}^{6} \quad \boldsymbol{v}_{c}=\frac{1}{m} \boldsymbol{S}_{v} \boldsymbol{h}_{c},
$$

where $\boldsymbol{p} \in \mathbb{R}^{3}$ and $\boldsymbol{l}_{c} \in \mathbb{R}^{3}$ are the linear momentum and the angular momentum around the CoM, respectively, $m \in \mathbb{R}$ is the total mass, and $\boldsymbol{S}_{v}=\left[\begin{array}{ll}\boldsymbol{E} & \mathbf{0}\end{array}\right] \in \mathbb{R}^{3 \times 6}$. The symbol $\boldsymbol{E}$ denotes the identity matrix of suitable dimensions. The momentum is computed based on generalized velocities, as

$$
\boldsymbol{h}_{c}=\boldsymbol{L}\left(\boldsymbol{R}_{b}, \boldsymbol{q}\right)\left[\begin{array}{c}
\boldsymbol{\nu}_{b} \\
\dot{\boldsymbol{q}}
\end{array}\right]
$$


where $\boldsymbol{L}\left(\boldsymbol{R}_{b}, \boldsymbol{q}\right)=\boldsymbol{A} \boldsymbol{d}_{c b}^{-T}\left(\boldsymbol{R}_{b}, \boldsymbol{q}\right)\left[\boldsymbol{M}_{b}(\boldsymbol{q}) \boldsymbol{M}_{b m}(\boldsymbol{q})\right]$. An average [15] velocity is associated to the momentum, as

$$
\boldsymbol{\nu}_{c}:=M_{c}\left(\boldsymbol{R}_{b}, \boldsymbol{q}\right)^{-1} \boldsymbol{h}_{c},
$$

where $\boldsymbol{M}_{c}\left(\boldsymbol{R}_{b}, \boldsymbol{q}\right)=\left(\boldsymbol{A d}_{c b}\left(\boldsymbol{R}_{b}, \boldsymbol{q}\right) \boldsymbol{M}_{b}(\boldsymbol{q})^{-1} \boldsymbol{A} \boldsymbol{d}_{c b}\left(\boldsymbol{R}_{b}, \boldsymbol{q}\right)^{T}\right)^{-1}$ is the inertia of the whole robot around the CoM. By using (5), the average velocity is related to generalized velocities, as

$$
\boldsymbol{\nu}_{c}=\boldsymbol{A} \boldsymbol{d}_{c b} \boldsymbol{\nu}_{b}+\boldsymbol{A} \boldsymbol{d}_{c b} \boldsymbol{M}_{b}^{-1} \boldsymbol{M}_{b m} \dot{\boldsymbol{q}} .
$$

Only external forces do change the momentum; thus it holds

$$
\dot{\boldsymbol{h}}_{c}=\boldsymbol{A d}_{c b}\left(\boldsymbol{R}_{b}, \boldsymbol{q}\right)^{-T} \mathcal{F}_{b}+\boldsymbol{A} \boldsymbol{d}_{c e}\left(\boldsymbol{R}_{b}, \boldsymbol{q}\right)^{-T} \mathcal{F}_{e}^{e x t} .
$$

Henceforth, the functional dependence is dropped out for the sake of brevity.

\section{Compliant FloAting-Base Control}

The objective of the control is to regulate the pose of the frame $\mathcal{E}$ around a constant, desired frame blue $\mathcal{E}_{d}$ while simultaneously satisfying requirements (R1),(R2),(R3). To ensure requirement (R3), a momentum end-effector task space and an external-internal actuation is employed. To ensure requirements (R1) and (R2), a compliant control design featuring end-effector compliance control and whole-body CoM and angular momentum regulation with force feedback is employed.

\section{A. Momentum-End Effector Task Space}

In order to design the controller at task level [16], the dynamics is transformed to a momentum end-effector task space. By using (2) and (5), a transformation is defined as

$$
\left[\begin{array}{c}
\boldsymbol{h}_{c} \\
\boldsymbol{\nu}_{e}
\end{array}\right]=\underbrace{\left[\begin{array}{cc}
\boldsymbol{A d}_{c b}^{-T} \boldsymbol{M}_{b} & \boldsymbol{A} \boldsymbol{d}_{c b}^{-T} \boldsymbol{M}_{b m} \\
\boldsymbol{A} \boldsymbol{d}_{e b} & \boldsymbol{J}_{e m}
\end{array}\right]}_{\boldsymbol{\Gamma}_{\mathrm{task}} \in \mathbb{R}^{12 \times(6+n)}}\left[\begin{array}{c}
\boldsymbol{\nu}_{b} \\
\dot{\boldsymbol{q}}
\end{array}\right] .
$$

In the letter, the control method is restricted to:

Assumption 1: The arm has six DOFs, i.e., $n=6$,

Based on Assumption 1, the task space is not redundant, and there is no remaining self motion. When $\boldsymbol{\Gamma}_{\text {task }}$ is not singular it is possible to invert (9); then, by pre-multiplying (3) by $\boldsymbol{\Gamma}_{\text {task }}^{T}$ and by inserting therein the inverse of (9) and its time derivative, the dynamics is transformed as

$$
\begin{aligned}
& \underbrace{\left[\begin{array}{cc}
\boldsymbol{M}_{h} & \boldsymbol{M}_{h e} \\
\boldsymbol{M}_{\text {he }}^{T} & \boldsymbol{M}_{e}
\end{array}\right]}_{\boldsymbol{M}_{\text {task }}}\left[\begin{array}{l}
\dot{\boldsymbol{h}}_{c} \\
\dot{\boldsymbol{\nu}}_{e}
\end{array}\right]+\underbrace{\left[\begin{array}{cc}
\boldsymbol{C}_{h} & \boldsymbol{C}_{h e} \\
\boldsymbol{C}_{e h} & \boldsymbol{C}_{e}
\end{array}\right]}_{\boldsymbol{C}_{\text {task }}}\left[\begin{array}{l}
\boldsymbol{h}_{c} \\
\boldsymbol{\nu}_{e}
\end{array}\right] \\
& =\boldsymbol{\Gamma}_{\text {task }}^{-T}\left[\begin{array}{c}
\mathcal{F}_{b} \\
\boldsymbol{\tau}
\end{array}\right]+\boldsymbol{\Gamma}_{\text {task }}^{-T} \boldsymbol{J}_{e}(\boldsymbol{q})^{T} \mathcal{F}_{e}^{\text {ext }},
\end{aligned}
$$

where the new dynamics matrices are given by

$$
\begin{aligned}
\boldsymbol{M}_{\text {task }} & =\boldsymbol{\Gamma}_{\text {task }}^{-T} \boldsymbol{M} \boldsymbol{\Gamma}_{\text {task }}^{-1}, \in \mathbb{R}^{12 \times 12} \\
\boldsymbol{C}_{\text {task }} & =\boldsymbol{\Gamma}_{\text {task }}^{-T}\left(\boldsymbol{C} \boldsymbol{\Gamma}_{\text {task }}^{-1}+\boldsymbol{M} \frac{d}{d t} \boldsymbol{\Gamma}_{\text {task }}^{-1}\right), \in \mathbb{R}^{12 \times 12}
\end{aligned}
$$

Equation (10) represents the so-called task-space dynamics [16] of the controller.

It allows to straightforwardly identify the couplings of the momentum in the end-effector dynamics while simultaneously ensuring the passivity property ${ }^{2}$ :

$$
\dot{M}_{e}=C_{e}+C_{e}^{T} .
$$

\section{B. External-Internal Actuation}

In the original task-space formulation [16], new control inputs are defined such that they are dual to the task motion. This would mean choosing new control inputs $\overline{\boldsymbol{\alpha}}_{c} \in \mathbb{R}^{6}$ and $\mathcal{F}_{e} \in \mathbb{R}^{6}$ so that they are allocated to the actuators by the transpose of the task-space transformation, as

$$
\left[\begin{array}{c}
\mathcal{F}_{b} \\
\boldsymbol{\tau}
\end{array}\right]=\underbrace{\left[\begin{array}{cc}
\boldsymbol{M}_{b} \boldsymbol{A} \boldsymbol{d}_{c b}^{-1} & \boldsymbol{A} \boldsymbol{d}_{e b}^{T} \\
\boldsymbol{M}_{b m}^{T} \boldsymbol{A} \boldsymbol{d}_{c b}^{-1} & \boldsymbol{J}_{\text {em }}^{T}
\end{array}\right]}_{\boldsymbol{\Gamma}_{\text {task }}^{T}}\left[\begin{array}{c}
\overline{\boldsymbol{\alpha}}_{c} \\
\mathcal{F}_{e}
\end{array}\right] .
$$

Then, the control task would be set on the new inputs $\overline{\boldsymbol{\alpha}}_{c}$ and $\mathcal{F}_{e}$. In our case this choice would be unfavorable because the map (13) is fully coupled and it would distribute the end-effector task both on the thrusters and on the joint torques. This in turn would imply that the thrusters be active during the pre-contact phase, thus disregarding requirement (R3).

A different allocation structure is used herein. More specifically, a so-called external/internal actuators allocation [7], [18] is employed. In such allocation, the manipulator control is decoupled from the thrusters input and does not excite the momentum of the system. An external motion is defined to be a 6DOF gross motion of the system; an internal motion is defined as a motion taking place in the dynamically-consistent null space of the momentum. We refer the reader to [7], [18] for a detailed description of the external/internal allocation; herein, we report it in a slightly modified form in terms of average velocity. Let us represent the external motion of the system by the average velocity $\boldsymbol{\nu}_{c}$. The so-called internal velocity of the end-effector [7] is given by

$$
\boldsymbol{\nu}_{e}^{i n t}:=\boldsymbol{J}_{e m}^{*} \dot{\boldsymbol{q}} \in \mathbb{R}^{6},
$$

where $\boldsymbol{J}_{e m}^{*}=\boldsymbol{J}_{e m}-\boldsymbol{A} \boldsymbol{d}_{e b} \boldsymbol{M}_{b}^{-1} \boldsymbol{M}_{b m}$ maps the joint velocity to the end-effector internal velocity, and is known in the literature as "generalized manipulator Jacobian" [12]. Note that by using (2), (5), (7), and (14) the end-effector velocity can be rewritten as sum of external and internal motions, as

$$
\boldsymbol{\nu}_{e}=\boldsymbol{A} \boldsymbol{d}_{e c} \boldsymbol{\nu}_{c}+\boldsymbol{\nu}_{e}^{\text {int }} .
$$

The external-internal allocation space is defined as

$$
\left[\begin{array}{c}
\boldsymbol{\nu}_{c} \\
\boldsymbol{\nu}_{e}^{i n t}
\end{array}\right]=\underbrace{\left[\begin{array}{cc}
\boldsymbol{A} \boldsymbol{d}_{c b} & \boldsymbol{A} \boldsymbol{d}_{c b} \boldsymbol{M}_{b}^{-1} \boldsymbol{M}_{b m} \\
\mathbf{0} & \boldsymbol{J}_{e m}^{*}
\end{array}\right]}_{\boldsymbol{\Gamma}_{\text {act }} \in \mathbb{R}^{12 \times(6+n)}}\left[\begin{array}{c}
\boldsymbol{\nu}_{b} \\
\dot{\boldsymbol{q}}
\end{array}\right] .
$$

Then, the external-internal actuators allocation is defined as

$$
\left[\begin{array}{c}
\mathcal{F}_{b} \\
\boldsymbol{\tau}
\end{array}\right]=\underbrace{\left[\begin{array}{cc}
\boldsymbol{A d}_{c b}^{T} & \mathbf{0} \\
\boldsymbol{M}_{b m}^{T} \boldsymbol{M}_{b}^{-1} \boldsymbol{A} \boldsymbol{d}_{c b}^{T} & \boldsymbol{J}_{\text {em }}^{* T}
\end{array}\right]}_{\boldsymbol{\Gamma}_{\mathrm{act}}^{T}}\left[\begin{array}{c}
\mathcal{F}_{c} \\
\mathcal{F}_{e}^{\text {int }}
\end{array}\right]
$$

\footnotetext{
${ }^{2}$ The passivity property is preserved [17, p.32], by the transformation (11), i.e., it holds $\dot{M}_{\text {task }}=\boldsymbol{C}_{\text {task }}+\boldsymbol{C}_{\text {task }}^{T}$. In turn, the property must hold also for each sub-block on their diagonal, thus yielding (12).
} 
where $\mathcal{F}_{c} \in \mathbb{R}^{6}, \mathcal{F}_{e}^{\text {int }} \in \mathbb{R}^{6}$ are new control inputs, which are dual to $\boldsymbol{\nu}_{c}, \boldsymbol{\nu}_{e}^{i n t}$. Notice in (17) that, in contrast to the task space allocation (13), the end effector control input $\mathcal{F}_{e}^{\text {int }}$ is only assigned to the joint torques, and not to the thrusters. This avoids activation of the thrusters during pre-contact end-effector maneuvering; thus, it contributes to satisfying (R3).

Let us now analyze the resulting form of the task-space dynamics under external/internal actuation. By inserting the first row of (17) in (8), it is obtained

$$
\dot{\boldsymbol{h}}_{c}=\mathcal{F}_{c}+\boldsymbol{A} \boldsymbol{d}_{c e}^{T} \mathcal{F}_{e}^{e x t} .
$$

By inserting (17) in (10), and by selecting the second row from the resulting expression, it is obtained ${ }^{3}$

$$
\boldsymbol{M}_{e} \dot{\boldsymbol{\nu}}_{e}+\boldsymbol{C}_{e} \boldsymbol{\nu}_{e}=-\boldsymbol{M}_{e h} \dot{\boldsymbol{h}}_{c}-\boldsymbol{C}_{e h} \boldsymbol{h}_{c}+\mathcal{F}_{e}^{i n t}+\mathcal{F}_{e}^{e x t} .
$$

The next section describes the control design based on the new inputs $\mathcal{F}_{c}$ and $\mathcal{F}_{e}^{\text {int }}$.

\section{Compliant Control Design}

To satisfy (R2), the controller must stabilize the entire system during contact. This is not achieved in the compliant floatingbase controllers [1], [2], [5], [8], [12]. In the proposed controller, it is achieved via feedback of the whole-body momentum and CoM, together with contact wrench feedback in the external controller. The external controller is

$$
\mathcal{F}_{c}=-\boldsymbol{S}_{v}^{T} \boldsymbol{K}_{c} \tilde{\boldsymbol{x}}_{c}-\boldsymbol{D}_{h} \boldsymbol{h}_{c}+\boldsymbol{A} \boldsymbol{d}_{e c}^{T} \mathcal{F}_{e}^{e x t}
$$

where $\tilde{\boldsymbol{x}}_{c}=\boldsymbol{o}_{c}-\boldsymbol{o}_{c_{d}} \in \mathbb{R}^{3}$ is the error of the CoM w.r.t. a constant, desired CoM position, $\boldsymbol{K}_{c} \in \mathbb{R}^{3 \times 3}$ is a symmetric, positive definite position gain matrix, and $\boldsymbol{D}_{h} \in \mathbb{R}^{6 \times 6}$ is a positive definite momentum gain matrix. Note that the whole-body momentum and CoM stabilization also allows the free motion of the base during pre-contact maneuvering, thereby achieving (R3). The controller of the end-effector is

$$
\mathcal{F}_{e}^{i n t}=-\boldsymbol{J}_{\tilde{x}_{e}}^{T} \boldsymbol{K}_{e} \tilde{\boldsymbol{x}}_{e}-\boldsymbol{D}_{e} \boldsymbol{\nu}_{e}
$$

where $\tilde{\boldsymbol{x}}_{e} \in \mathbb{R}^{6}$ are error coordinates for the end effector, $\boldsymbol{J}_{\tilde{x}_{e}} \in \mathbb{R}^{6 \times 6}$ is the coordinates Jacobian, $\boldsymbol{K}_{e} \in \mathbb{R}^{6 \times 6}$ is a symmetric, positive definite stiffness matrix, and $D_{e} \in \mathbb{R}^{6 \times 6}$ is a positive definite damping matrix. The exact expressions of $\tilde{\boldsymbol{x}}_{e}$ and $\boldsymbol{J}_{\tilde{x}_{e}}$ can be found in [18, Eq. (B.6b)]. Note that the cancellation of the contact wrench happens only in the external subsystem - and not in the end effector; this is what yields a compliant interaction and thus satisfaction of (R1). The time derivative of the errors is given by

$$
\begin{aligned}
& \dot{\tilde{\boldsymbol{x}}}_{c}=\boldsymbol{v}_{c}=\frac{1}{m} \boldsymbol{S}_{v} \boldsymbol{h}_{c}, \\
& \dot{\tilde{\boldsymbol{x}}}_{e}=\boldsymbol{J}_{\tilde{x}_{e}} \boldsymbol{\nu}_{e} .
\end{aligned}
$$

where (4) is applied. The closed-loop dynamics can be written [8] in state-space form as

$$
\dot{z}=\boldsymbol{f}(\boldsymbol{z}), \quad \boldsymbol{z}=\left[\begin{array}{lllll}
\boldsymbol{h}_{c}^{T} & \tilde{\boldsymbol{x}}_{c}^{T} & \boldsymbol{\nu}_{e}^{T} & \tilde{\boldsymbol{x}}_{e}^{T} & \boldsymbol{q}^{T}
\end{array}\right]^{T} \in D \subset \mathbb{R}^{21+n} .
$$

\footnotetext{
${ }^{3}$ The simplification of the wrenches-related terms in (18b) follows from straightforward application of the block-inverse of (9), yielding $\left[\begin{array}{ll}\mathbf{0} & \boldsymbol{E}\end{array}\right] \boldsymbol{\Gamma}_{\text {task }}^{-T} \boldsymbol{\Gamma}_{a c t}^{T}=\boldsymbol{E}$ and $\left[\begin{array}{ll}\mathbf{0} & \boldsymbol{E}\end{array}\right] \boldsymbol{\Gamma}_{\text {task }}^{-T} \boldsymbol{J}_{e}^{T}=\boldsymbol{E}$.
}

Proposition III.1 (Global exponential stability of momentum and $\mathrm{CoM}$ ): The equilibrium $\boldsymbol{h}_{c}=\mathbf{0}, \tilde{\boldsymbol{x}}_{c}=\mathbf{0}$ of the subsystem (18a), (19a), (20a), is globally exponentially stable.

Proof: The stability is proven with the Lyapunov function

$$
V_{h}=\frac{1}{2} m \boldsymbol{v}_{c}^{T} \boldsymbol{v}_{c}+\frac{1}{2} \boldsymbol{l}_{c}^{T} \boldsymbol{l}_{c}+\frac{1}{2} \tilde{\boldsymbol{x}}_{c}^{T} \boldsymbol{K}_{c} \tilde{\boldsymbol{x}}_{c}
$$

which is radially unbounded. By using (4), (18a), (19a) and (20a), its time derivative is $\dot{V}_{h}=-\boldsymbol{h}_{c}^{T} \boldsymbol{D}_{h} \boldsymbol{h}_{c}$, which is negative semi-definite. By applying LaSalle, it results $\tilde{\boldsymbol{x}}_{c} \rightarrow \mathbf{0}$ and thus the global asymptotic stability is proven. Because the (18a), (19a) and (20a) subsystem is linear, the equilibrium is globally exponentially stable.

Proposition III.2 (Passivity): The subsystem (18b), (19b), and (20b) is passive w.r.t. the input-output pair $\mathcal{F}_{e}^{e x t}-\boldsymbol{M}_{e h} \dot{\boldsymbol{h}}_{c}-\boldsymbol{C}_{e h} \boldsymbol{h}_{c} \rightarrow \boldsymbol{\nu}_{e}$.

Proof: Let us consider the storage function

$$
S=V_{h}+\frac{1}{2} \boldsymbol{\nu}_{e}^{T} \boldsymbol{M}_{e} \boldsymbol{\nu}_{e}+\frac{1}{2} \tilde{\boldsymbol{x}}_{e}^{T} \boldsymbol{K}_{e} \tilde{\boldsymbol{x}}_{e} .
$$

Its time derivative is given by

$$
\begin{aligned}
\dot{S} & =-\boldsymbol{h}_{c}^{T} \boldsymbol{D}_{h} \boldsymbol{h}_{c}-\boldsymbol{\nu}_{e}^{T} \boldsymbol{D}_{e} \boldsymbol{\nu}_{e}+\boldsymbol{\nu}_{e}^{T}\left(\mathcal{F}_{e}^{e x t}-\boldsymbol{M}_{e h} \dot{\boldsymbol{h}}_{c}-\boldsymbol{C}_{e h} \boldsymbol{h}_{c}\right) \\
& \leq \boldsymbol{\nu}_{e}^{T}\left(\mathcal{F}_{e}^{e x t}-\boldsymbol{M}_{e h} \dot{\boldsymbol{h}}_{c}-\boldsymbol{C}_{e h} \boldsymbol{h}_{c}\right),
\end{aligned}
$$

where (18b), (19b), (20b), and the property (12), are used. Based on (24), the subsystem is proven passive.

Based on Prop.III.1, it is concluded that also the requirement (R2) is achieved. From Prop.III.2 it is concluded that after the momentum converges the arm behaves passively w.r.t the physical variables $\mathcal{F}_{e}^{\text {ext }}$ and $\boldsymbol{\nu}_{e}$. In presence of a constant external task wrench, the whole system has a set of equilibria given by $\boldsymbol{z}_{0}=\left[\begin{array}{lllll}\mathbf{0}^{T} & \mathbf{0}^{T} & \mathbf{0}^{T} & \boldsymbol{x}_{e}^{* T} & \boldsymbol{q}^{T}\end{array}\right]^{T}$, where $\tilde{\boldsymbol{x}}_{e}^{* T} \in \mathbb{R}^{6}$ is the constant, displaced equilibrium position of the end-effector stiffness in presence of constant wrench, given by

$$
\boldsymbol{x}_{e}^{*}=\boldsymbol{K}_{e}^{-1} \overline{\mathcal{F}}_{e}^{e x t},
$$

where $\overline{\mathcal{F}}_{e}^{\text {ext }}=\boldsymbol{J}_{\tilde{x}_{e}}^{-T} \mathcal{F}_{e}^{e x t}$ is the task wrench.

Proposition III.3 (Asymptotic stability of whole system): During steady contact, i.e., $\overline{\mathcal{F}}_{e}^{\text {ext }}=$ const, the set of equilibria $\boldsymbol{z}=\boldsymbol{z}_{0}$ of the system (21) is asymptotically stable.

Proof: The set of equilibria $\boldsymbol{z}_{0}$ is compact because the joint space is compact. The stability is addressed in two steps in a hierarchical fashion, proving first the asymptotic stability of the CoM-momentum subsystem, and then the asymptotic stability of the rest of the system in the subset of converged momentum $\Omega=\left\{\boldsymbol{z} \in D: \boldsymbol{h}_{c}=\mathbf{0}, \tilde{\boldsymbol{x}}_{c}=\mathbf{0}\right\}$. The asymptotic stability of the CoM-momentum subsystem is already proven by Prop.III.1. Regarding the stability of the rest of the system in $\Omega$, the Lyapunov function candidate

$$
V_{e}=\frac{1}{2} \boldsymbol{\nu}_{e}^{T} \boldsymbol{M}_{e} \boldsymbol{\nu}_{e}+\frac{1}{2}\left(\tilde{\boldsymbol{x}}_{e}-\boldsymbol{x}_{e}^{*}\right)^{T} \boldsymbol{K}_{e}\left(\tilde{\boldsymbol{x}}_{e}-\boldsymbol{x}_{e}^{*}\right)
$$

has time derivative

$$
\begin{aligned}
\dot{V}_{e} & =\boldsymbol{\nu}_{e}^{T} \boldsymbol{M}_{e} \dot{\boldsymbol{\nu}}_{e}+\frac{1}{2} \boldsymbol{\nu}_{e}^{T} \dot{\boldsymbol{M}}_{e} \boldsymbol{\nu}_{e}+\dot{\tilde{\boldsymbol{x}}}_{e}^{T} \boldsymbol{K}_{e}\left(\tilde{\boldsymbol{x}}_{e}-\boldsymbol{x}_{e}^{*}\right) \\
& =-\boldsymbol{\nu}_{e}^{T} \boldsymbol{D}_{e} \boldsymbol{\nu}_{e}+\frac{1}{2} \boldsymbol{\nu}_{e}^{T}\left(\dot{\boldsymbol{M}}_{e}-2 \boldsymbol{C}_{e}\right) \boldsymbol{\nu}_{e}+\boldsymbol{\nu}_{e}^{T} \boldsymbol{J}_{\tilde{x}_{e}}^{T}\left(\overline{\mathcal{F}}_{e}^{e x t}-\boldsymbol{K}_{e} \boldsymbol{x}_{e}^{*}\right) \\
& =-\boldsymbol{\nu}_{e}^{T} \boldsymbol{D}_{e} \boldsymbol{\nu}_{e} \leq 0
\end{aligned}
$$


where (12), (18b), (19b), (20b), (25), and $\boldsymbol{h}_{c} \equiv \mathbf{0}$ are used. Thus, $z_{0}$ is stable relative to [19] $\Omega$. By applying LaSalle in $\Omega$, it holds $\boldsymbol{\nu}_{e} \equiv \mathbf{0} \Rightarrow \tilde{\boldsymbol{x}}_{e}=\boldsymbol{x}_{e}^{*}$, thus $\boldsymbol{z}_{0}$ is asymptotically stable relative to $\Omega$. Then, $\boldsymbol{z}_{0}$ is asymptotically stable by hierarchical asymptotic stability [19].

Conclusively, the controller meets all requirements (R1)(R3). Next, its validation is shown.

\section{NUMERICAL VALIDATION}

Two numerical simulations are carried out in order to analyze and validate the control method. In the first one, the performance of two state-of-the-art controllers is reported and their limitations are underlined. In the second one, the effectiveness of the proposed controller in solving those limitations is shown, as well as its applicability considering models of discrete thrusters and noisy measurements.

A representative capture operation was simulated, which involved contact-free maneuvering of the end-effector as well as a contact phase. The end effector was commanded to reach a desired pose consisting in a displacement of $\Delta \boldsymbol{o}_{e}=\left[\begin{array}{lll}18 & -16 & -18\end{array}\right] \mathrm{cm}$ in translation and $\Delta \phi_{e}=\left[\begin{array}{lll}-2 & -2 & 9\end{array}\right]$ deg in rotation ${ }^{4}$, from the initial pose. A $10 \mathrm{~s}$ end-effector trajectory was commanded to the robot, it was generated using a smooth 5th order interpolator. The $\mathrm{CoM}$ of the system is commanded to stay in its initial position. The simulated arm has six DOFs, and consists in a KUKA KR4+ lightweight robot with locked third joint, which weights approximately $17 \mathrm{~kg}$. The dynamics parameters for the spacecraft are: mass $150 \mathrm{~kg}$, inertia blkdiag $(21.8,15,18.88) \mathrm{kgm}^{2}$. At $t_{1}=5 \mathrm{~s}$, a steady contact ${ }_{I} \boldsymbol{f}_{e}^{\text {ext }}=\left[\begin{array}{lll}-10 & 0 & 0\end{array}\right]^{T} \mathrm{~N}$, ${ }_{I} \boldsymbol{\tau}_{e}^{e x t}=\left[\begin{array}{lll}0 & 0 & 0\end{array}\right]^{T} \mathrm{Nm}$ is ${ }^{5}$ given to the end effector. The stiffness gains were $\boldsymbol{K}_{e}=\operatorname{blkdiag}\left(\boldsymbol{K}_{e, t r a s l}, \boldsymbol{K}_{e, \text { rot }}\right)$ for the end effector, where $\boldsymbol{K}_{e, t r a s l}=1000 \boldsymbol{E} \mathrm{Nm}^{-1}, \boldsymbol{K}_{e, \text { rot }}=$ $70 \boldsymbol{E} \mathrm{Nm} \mathrm{rad}^{-1}$, and $\boldsymbol{K}_{c}=128 \boldsymbol{E} \mathrm{Nm}^{-1}$ for the CoM, respectively. The $\mathrm{EE}$ and $\mathrm{CoM}$ damping gains were designed using the method in [17, p.36] based on the inertia in the initial configuration. The angular momentum gain was $\boldsymbol{D}_{l}=1 \boldsymbol{E} \mathrm{s}^{-1}$.

\section{A. Simulation of State-of-the-art Controllers}

The proposed controller is compared with the following stateof-the-art compliant controllers considering ideal thrusters and measurements:

(C1) Free-floating + switched thrusters activation [12]

(C2) Floating-Base via CoM regulation and angular momentum dumping [8]

In (C1) the robot is controlled in free-floating mode during the pre-contact phase; once contact happens, thrusters are activated to control the distance between the spacecraft and the target around a desired value. The end effector's compliant behavior is enforced via feedback linearization.

In (C2) the spacecraft pose is not controlled - similarly as in (C1) - but the thrusters are employed to regulate the whole-body $\mathrm{CoM}$ and angular momentum of the robot. The controller works as a free-floating during the pre-contact phase and automatically

\footnotetext{
${ }^{4} \phi_{e} \in \mathbb{R}^{3}$ denotes the array vector of roll, pitch, yaw angles of the end effector; they are not used in the controller, but only for visualization.

${ }^{5}$ The left subscript in ${ }_{I} \boldsymbol{f}_{e}^{\text {ext }}$ and ${ }_{I} \boldsymbol{\tau}_{e}^{\text {ext }}$ means that the quantities are expressed in inertial axes.
}

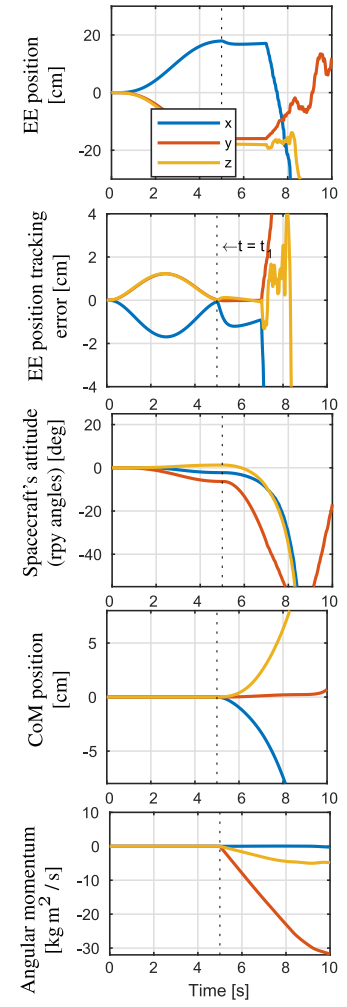

(C1)
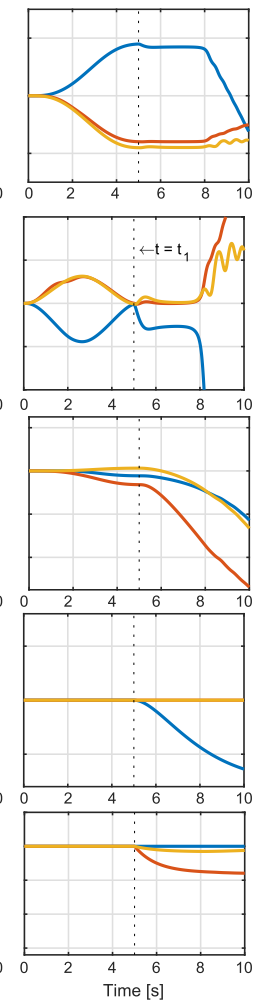

(C2)
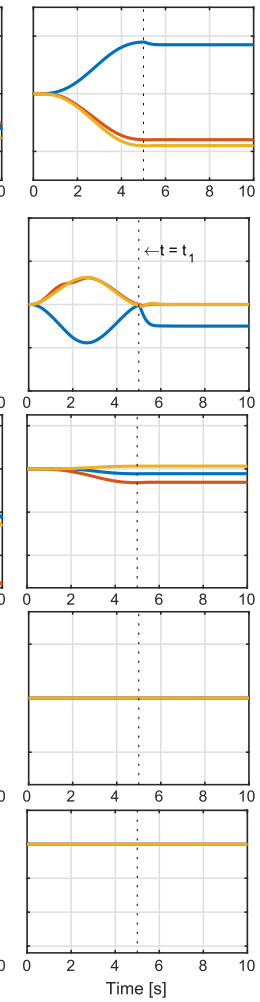

(proposed)
Fig. 2. Comparison of state-of-the-art and proposed controllers considering ideal thrusters and measurements.

fires the thrusters after contact; this is accomplished by a single continuous controller without the need of switching as in (C1). In (C2), the end effector compliant behavior is realized by a task-space PD control law, and the thrusters command is given by

$$
\mathcal{F}_{b}=\boldsymbol{A d}_{c b}^{T}\left(-\boldsymbol{S}_{v}^{T} \boldsymbol{K}_{c} \tilde{\boldsymbol{x}}_{c}-\boldsymbol{D}_{h} \boldsymbol{h}_{c}\right)
$$

Fig. 2 shows the simulation results of the state-of-art controllers, as well as of the proposed one. In Fig. 2, the end effector position and tracking error, the spacecraft's attitude, the wholebody CoM position and angular momentum, the commanded spacecraft force and torque, are shown; the start of the contact is signaled in the figure by means of a vertical dotted line; the position and angles are w. r. t. initial values.

Regarding (C1), Fig. 2 shows that the end effector was effectively steered towards the desired pose. Right at the end of the maneuver, a steady contact happened and a compliant behavior of the end effector was achieved for approximately two seconds. However, an instability happened in the mean time in the rest of the system, as it is clearly seen in the plots of the spacecraft's attitude, as well as in those of the CoM and angular momentum. This instability was due to the lack of actuation on two translation DOFs and three rotational DOFs; it promptly led to singularity due to stretch out of the arm, and, in turn, to a failure of the end effector task.

Regarding (C2), Fig. 2 shows that the end effector was effectively steered towards the desired location, and that a compliant displacement of the end effector was realized in contact. A drift happened in the rest of the system. Differently than (C1), the 

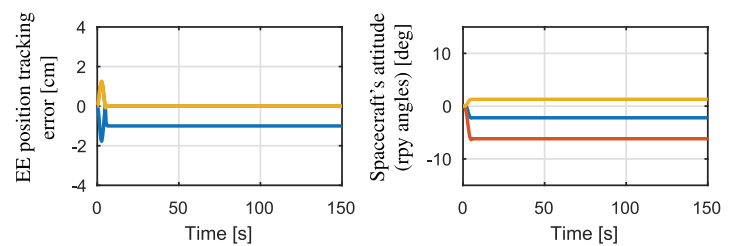

Fig. 3. Longer simulation of proposed controller.

unbounded growth was slower and happening at a bounded rate; further, it happened only in the spacecraft's rotation. However, this drift was enough to compromise the end effector task within approximately $3 \mathrm{~s}$. This drift is explained by the nonzero steadystate angular momentum during contact, which originates by the use of only a damping action in the angular momentum controller. Regarding the CoM, in Fig. 2 it can be observed that during contact it reached a displaced value. This displacement contributes to the stretch out of the arm and, in presence of gains compatible with realistic thrusters, may be high. The angular drift and CoM displacement during contact are considered the two limitations of controller (C2).

Regarding the proposed controller, Fig. 2 shows that it is successful in steering the end effector towards the desired location, and in achieving a compliant interaction in contact. Importantly, in contrast to the state-of-the-art controllers, they also show that the proposed controller is not affected by drift, i.e., the attitude reaches a fixed value during the steady contact. In spite of the stable response, the proposed controller could be simulated for a longer time, and the results are shown in Fig. 3; they confirm the absence of attitude drift, and that the controller can sustain contact for undefinite contact. Thus, it is concluded that the proposed controller solves the limitations of the state-of-the-art controllers, and effectively achieves end-effector maneuvering and stable compliant contact in presence of long contact.

\section{B. Simulation of Proposed Controller in Presence of Thrusters and Noise Models}

The scope of this section is to validate the proposed controller works well in presence of realistic models of thrusters and noise. The same maneuver and contact as in the previous simulation have been used. In this simulation the contact was removed after a certain time $t_{2}$ to show also post-contact response. A redundant thrusters system composed of 24 thrusters is modeled, which provides full actuation capability on all translational and rotational components. All thrusters are the same and have a thrust of $5 \mathrm{~N}$ and a Minimum Impulse Bit (MIB) of $0.05 \mathrm{~N}$ s. The satellite control signal $\mathcal{F}_{b}$ is sampled at a $300 \mathrm{~ms}$ rate, whereas the joint control signal $\tau_{m}$ is sampled at a $1 \mathrm{~ms}$ rate. More information about the thrusters model can be found in [20]. A Gaussian noise with zero mean is used to model the uncertainties in the joint, spacecraft, and end-effector measurements; the standard deviation and sampling rate are in [18, Table 7.3]. The whole-body CoM and momentum states are reconstructed based on such noisy measurements and on the inertia model [18, Sect 2.2.2]. A time delay of $50 \mathrm{~ms}$ and Gaussian noise of $0.2 \mathrm{~N}$ and $0.2 \mathrm{Nm}$ standard deviation on the external force and torque measurement was simulated to account for the effects of a realistic force sensor or estimator. A first-order filter with $15 \mathrm{~Hz}$ cut-off frequency and a deadzone of $\pm 2.4 N$ in translation and
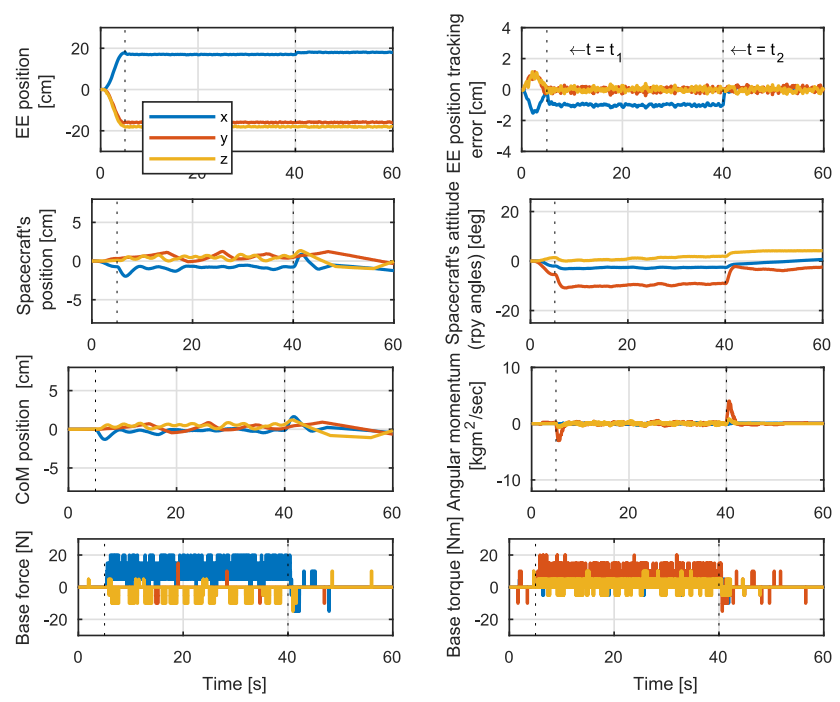

Fig. 4. Simulation of proposed controller with realistic thrusters and noisy measurements.

$\pm 0.2 \mathrm{Nm}$ in rotation, are used on thrusters commands. A disturbance torque $\boldsymbol{\tau}_{b, d}=[1 ; 1 ; 1] \cdot 10^{-4} \mathrm{Nm}$ around the base was simulated to model the effects of environmental disturbances in orbit.

Fig. 4 shows the results. In Fig. 4, the plots of spacecraft's position, and of the thrusters are additionally shown. First, notice in Fig. 4 that the thrusters force and torque show a pulsed behavior. Even in the presence of realistic thrusters and noise, the controller achieves the desired objectives, i.e., the end effector is steered towards the desired location and the contact takes place compliantly. Further, the attitude drift is successfully removed up to the resolution of the thrusters and noise models. The resulting wandering behavior of the spacecraft attitude is interpreted as being the main effect of such uncertainties; it is due to thrusters MIB, control deadzone, measurement noise, and environmental disturbances, and it is not present in ideal conditions, as clearly evident in the attitude plot in Fig. 3. The results clearly show that the control method works well in presence of realistic models of thrusters, measurement noise and time delay of the force measurement, thereby validating its applicability in realistic scenarios. Last but not least, they clearly show that the proposed controller is stable during pre-contact, contact, and post-contact phases, and that the thrusters are automatically switched on and off by a single continuous controller without recurring to control law switching.

\section{EXPERIMENTAL VALIDATION}

The proposed controller was validated on the On-Orbit Servicing Simulator (OOS-Sim) hardware-in-the-loop simulator [21] at the DLR (see Fig. 5). The space robot simulation consists in a test arm mounted on a spacecraft simulator arm. The simulator arm is an admittance-controlled KUKA KR120 industrial arm and is devoted to reproducing the spacecraft's dynamics based on a real-time model integration. The test arm is a torque-controlled KUKA KR4+ lightweight robot with seven DOFs. The microgravity conditions in the test arm are replicated by actively compensating the joint gravity torques based on an identified model. With this system, the space robot controller is simulated 


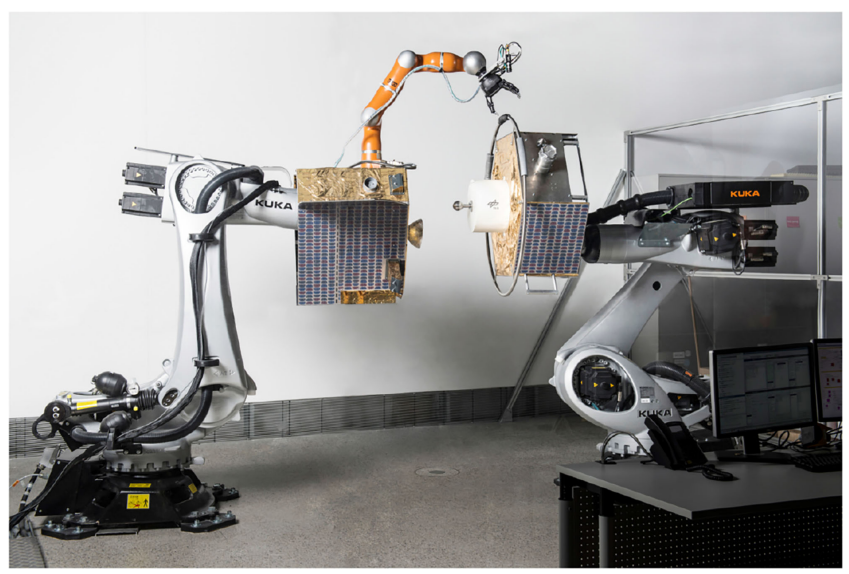

Fig. 5. The On-Orbit Servicing Simulator at DLR RMC.

taking into account real dynamics, sensors, time delay, control discretization, and model uncertainties of the test manipulator, whereas the spacecraft simulation is based on a model; the motion yielded by the model is then commanded to the simulator arm. The simulated spacecraft's mass and inertia are $300 \mathrm{~kg}$ and blkdiag $(43.67,29.92,37.75) \mathrm{kgm}^{2}$, respectively; no discrete thrusters are modelled in the experiments. More information about the facility can be found in [21].

\section{A. Controller Implementation}

The actuator commands are sampled at $1 \mathrm{~ms}$ time step; the model of the inertia is sampled at $10 \mathrm{~ms}$ time step. In the present test, the state is reconstructed using the forward kinematics of the test and simulator arms; the external wrench is approximately reconstructed using joint torque sensors [18, Chapt. 8.1]. In a space robot, the state can be reconstructed using an observer [22], [23]. The external wrench is measurable by an end effector-mounted force torque sensor or by an observer [24], [25]. The singularity of the controller corresponds to the singularities of the generalized Jacobian $\boldsymbol{J}_{e m}^{*}$ and can be avoided via trajectory planning [26].

\section{B. Experimental Results}

Two experiments are conducted. First, a sequence of contacts along different directions was given onto the end effector, to validate the contact response. The contacts are given manually by using a rod. Second, a contact-free maneuver of the end effector was commanded, to validate the controller during the pre-contact phase. The maneuver consisted in an end effector displacement of $\Delta \boldsymbol{o}_{e}=\left[\begin{array}{llll}12.8 & 0 & -4\end{array}\right] \mathrm{cm}$ in translation and $\Delta \phi_{e}=\left[\begin{array}{lll}0 & -10 & 0\end{array}\right] \mathrm{deg}$ in rotation, from the initial pose, followed by a return to the initial pose. In both experiments, the CoM was commanded to keep the initial location. In the maneuvering experiment, a simple model of thrusters MIB was simulated, consisting in a deadzone of $0.5 \mathrm{~N}$ on $f_{b}$ and of $0.5 \mathrm{Nm}$ on $\boldsymbol{\tau}_{b}$. The EE stiffness used in the contact experiment are $\boldsymbol{K}_{e, \text { trasl }}=500 \boldsymbol{E} \mathrm{Nm}^{-1}, \boldsymbol{K}_{e, \text { rot }}=35 \boldsymbol{E} \mathrm{Nm} \mathrm{rad}^{-1}$ for the contact experiment, and $\boldsymbol{K}_{e, t r a s l}=700 \boldsymbol{E} \mathrm{Nm}^{-1}, \boldsymbol{K}_{e, r o t}=$ $49 \boldsymbol{E ~} \mathrm{Nm} \mathrm{rad}^{-1}$ for the maneuvering experiment. The experiments can be seen in the attached video.

Fig. 6 shows the experimental results of the contact experiment; the figure shows the contact force and torque, the position
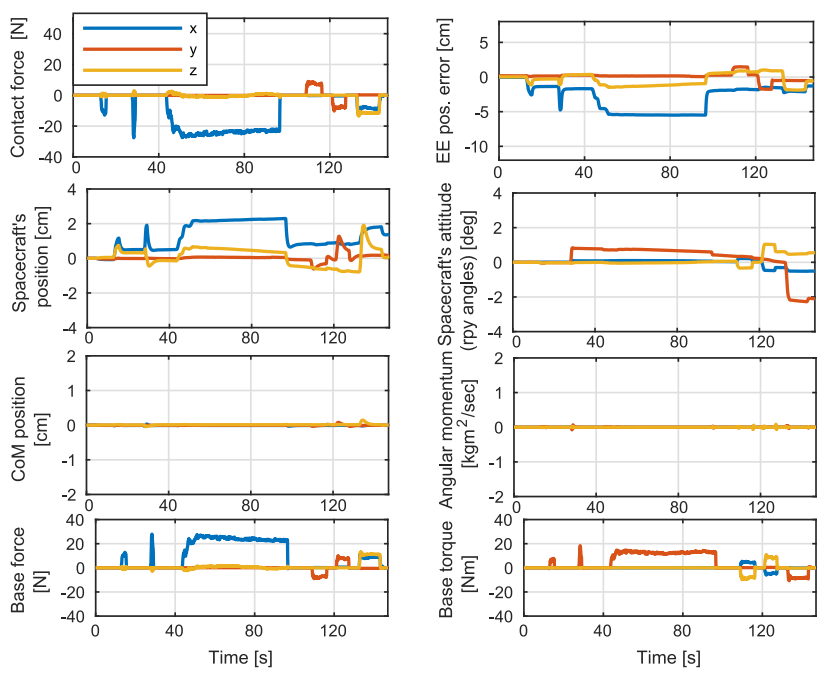

Fig. 6. Experimental response to contact.
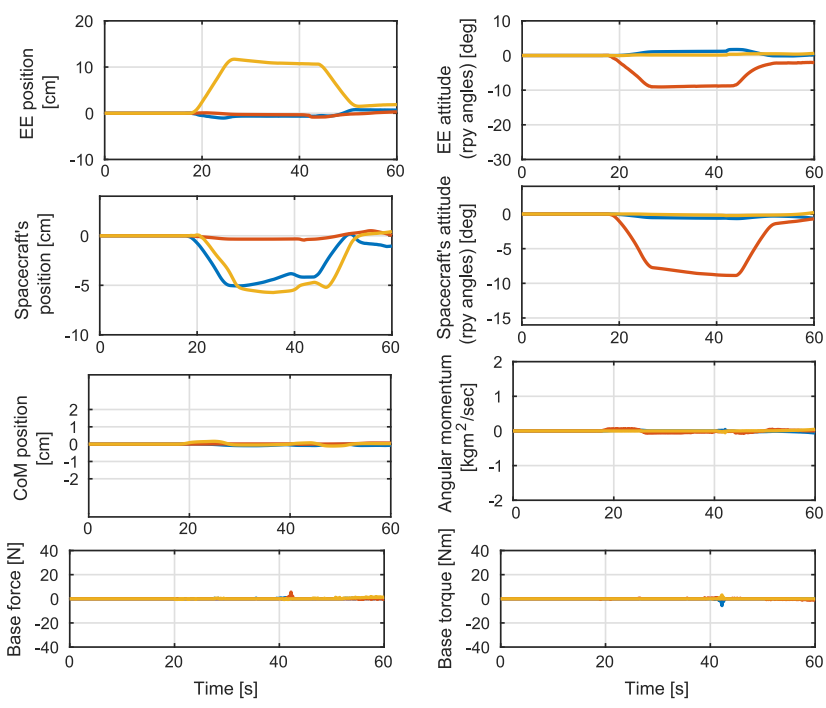

Fig. 7. Experimental response to end-effector maneuvering.

and attitude of the spacecraft, the position of the whole-body $\mathrm{CoM}$ position and angular momentum, and the thrusters force and torque. The first two contacts were impulsive, the third one was a very long contact along $\mathrm{x}$, and the last three ones were long contacts along other directions. In Fig. 6 it can be observed that the system behaved stably during and after each contact, and that a compliant interaction was achieved. The compliant interaction can be observed in particular in the plot of the end effector error, which displaced compliantly, and yielded a displacement compatible with the designed end effector stiffness up to small deviations due to the joint static friction. Fig. 6 further shows that the $\mathrm{CoM}$ and angular momentum were regulated to almost zero, and that no drift happened, up to the accuracy achievable with sensor noise and offsets in the facility, thereby confirming the advantage of the proposed control.

Fig. 7 shows the experimental results of the contact-free maneuvering experiment; the figure shows the end effector position and attitude, as well as similar plots as for the contact experiment. Observe in Fig. 7 how both the end-effector position and attitude were successfully steered to the desired position. 
Further, observe in Fig. 7 that the spacecraft displaced to new, stable locations after maneuvering. Finally, observe that the controller avoided any thrusters activation - thereby efficiently accomplishing the pre-contact maneuvering in a free-floating fashion, as envisioned in requirement (R3) -, apart from small spikes, which were due to noise spikes overcoming the thrusters MIB and can be mitigated by a suitable deadzone. The experiment confirmed the effectiveness of the control for pre-contact maneuvering. In both experiments, the self motion of the sevenDOFs arm was promptly stopped by the joint friction, thus no effects on the joint and base motion due to self motion were observed; in different situations in which control of the self motion is necessary, e.g., fast end-effector maneuvering, the controller can be extended similarly as in [18, Chapt 5.1]. In conclusion, the experiments confirmed the applicability of the proposed controller on real hardware.

\section{LIMITATIONS AND FUTURE WORK}

The proposed control method is successful in eliminating the instability and the drift of state-of-the-art compliant control strategies. In presence of thrusters, measurement noise, and orbital disturbances, a slight wandering of the attitude of the spacecraft still happens, which, despite being small in magnitude, may still lead to an overall unfavorable location after a certain time. We point out that this slight wandering does not hinder application to realistic floating-base missions, for which an attitude displacement is $\pm 30 \mathrm{deg}$ is acceptable [2]. However, to guarantee safety, future works may employ reaction wheels to absorb the residual momentum, or, in their absence, may extend the controller with an additional proportional action to enforce a box-type requirement on the attitude. Further, future works may validate experimentally the entire sequence of pre-contact, contact, post-contact phases.

\section{CONCLUSION}

A compliant floating-base controller was proposed. Regulation of whole-body CoM and angular momentum together with force feedback was employed in order to stabilize the system during general contact, thereby solving the instability and the drift limitations of state-of-the-art strategies. The method was validated via extensive experimental and numerical validation; it was shown that it can be applied on a real manipulator, and in presence of realistic thrusters, measurement noise, and time delay of the external force measurement. The controller is applicable to pre-contact, contact, and post-contact phases of a robotic operation.

\section{REFERENCES}

[1] Y. Masutani, F. Miyazaki, and S. Arimoto, "Modeling and sensory feedback control for space manipulators," in Proc. NASA Conf. Telerobotics, 1989, pp. 1346-1351.

[2] R. Lampariello, H. Mishra, N. Oumer, P. Schmidt, M. De Stefano, and A. Albu-Schäffer, "Tracking control for the grasping of a tumbling satellite with a free-floating robot," IEEE Robot. Automat. Lett., vol. 3, no. 4, pp. 3638-3645, Oct. 2018.
[3] K. Yoshida, N. Sashida, R. Kurazume, and Y. Umetani, "Modeling of collision dynamics for space free-floating links with extended generalized inertia tensor," in Proc. IEEE Int. Conf. Robot. Automat., 1992, pp. 899-904 vol. 1.

[4] D. N. Nenchev and K. Yoshida, "Impact analysis and post-impact motion control issues of a free-floating space robot contacting a tumbling object," Proc. IEEE Int. Conf. Robot. Automat., 1998, pp. 913-919 vol. 1.

[5] K. Yoshida et al., "Dynamics, control and impedance matching for robotic capture of a non-cooperative satellite," Adv. Robot., vol. 18, no. 2, pp. 175-198, 2004.

[6] K. Yoshida, D. Dimitrov, and H. Nakanishi, "On the capture of tumbling satellite by a space robot," in Proc. IEEE/RSJ Int. Conf. Intell. Robots Syst., 2006, pp. 4127-4132.

[7] A. M. Giordano, G. Garofalo, and A. Albu-Schäffer, "Momentum dumping for space robots," in Proc. IEEE 56th Annu. Conf. Decis. Control, 2017, pp. 5243-5248.

[8] A. M. Giordano, D. Calzolari, and A. Albu-Schäffer, "Workspace fixation for free-floating space robot operations," in Proc. IEEE Int. Conf. Robot. Automat., 2018, pp. 889-896.

[9] N. Uyama, H. Nakanishi, K. Nagaoka, and K. Yoshida, "Impedance-based contact control of a free-flying space robot with a compliant wrist for non-cooperative satellite capture," in Proc. IEEE/RSJ Int. Conf. Intell. Robots Syst., 2012, pp. 4477-4482.

[10] A. Stolfi, P. Gasbarri, and M. Sabatini, "A parametric analysis of a controlled deployable space manipulator for capturing a non-cooperative flexible satellite," Acta Astronautica, vol. 148, pp. 317-326, 2018.

[11] K. Nanos, F. Xydi-Chrysafi, and E. Papadopoulos, "On impact de-orbiting for satellites using a prescribed impedance behavior*," in Proc. IEEE 58th Conf. Decis. Control, 2019, pp. 2126-2131.

[12] H. Nakanishi and K. Yoshida, "Impedance control for free-flying space robots -basic equations and applications," in Proc. IEEE/RSJ Int. Conf. Intell. Robots Syst., 2006, pp. 3137-3142.

[13] R. M. Murray, Z. Li, and S. S. Sastry, A Mathematical Introduction to Robotic Manipulation, 1st ed. Boca Raton, FL, USA: CRC Press, 1994.

[14] G. Garofalo, C. Ott, and A. Albu-Schäffer, "On the closed form computation of the dynamic matrices and their differentiations," in Proc. IEEE/RSJ Int. Conf. Intell. Robots Syst., 2013, pp. 2364-2359.

[15] A. Saccon, S. Traversaro, F. Nori, and H. Nijmeijer, "On Centroidal dynamics and integrability of average angular velocity," IEEE Robot. Automat. Lett., vol. 2, no. 2, pp. 943-s950, Apr. 2017.

[16] O. Khatib, "A unified approach for motion and force control of robot manipulators: The operational space formulation," IEEE J. Robot. Automat., vol. 3, no. 1, pp. 43-53, Feb. 1987.

[17] C. Ott, Cartesian Impedance Control of Redundant and Flexible-Joint Robots. Berlin, Germany: Springer, 2008.

[18] A. M. Giordano, "Whole-body control of orbital robots," Ph.D. dissertation, Technische Universität München, 2020.

[19] M. I. El-Hawwary and M. Maggiore, "Reduction theorems for stability of closed sets with application to backstepping control design," Automatica, vol. 49, no. 1, pp. 214-222, 2013.

[20] A. M. Giordano et al., "Coordination of thrusters, reaction wheels, and arm in orbital robots," Robot. Auton. Syst., vol. 131, 2020, Art. no. 103564.

[21] J. Artigas et al., "The OOS-SIM: An on-ground simulation facility for on-orbit servicing robotic operations," in Proc. IEEE Int. Conf. Robot. Automat., 2015, pp. 2854-s2860.

[22] A. Gallardo et al., "Robust estimation of motion states for free-floating tumbling target capture," in Proc. IEEE Aerosp. Conf., 2019, pp. 1-11.

[23] H. Mishra, M. De Stefano, A. M. Giordano, and C. Ott, "A nonlinear observer for free-floating target motion using only pose measurements," in Proc. Annu. Amer. Control Conf., 2019, pp. 1114-1121.

[24] F. Cavenago et al., "Contact detection, isolation and estimation for orbital robots through an observer based on a centroid-joints dynamics," Acta Astronautica, vol. 181, pp. 40-51, 2021.

[25] F. Cavenago et al., "Unexpected collision detection, estimation, and reaction for a free-flying orbital robot," J. Guidance, Control, Dyn., vol. 44, no. 5, pp. 967-982, 2021.

[26] D. Calzolari, R. Lampariello, and A. M. Giordano, "Singularity maps of space robots and their application to gradient-based trajectory planning," in Robot.: Sci. Syst., Jul. 2020. 